\title{
Incidencia del Estado de Alarma en la Desigualdad Escolar de la Adolescencia Acogida por el Sistema de Protección en Galicia
}

\author{
Incidence of the State of Alarm on School Inequality of \\ Adolescents within the Protection System in Galicia
}

\author{
Deibe Fernández-Simo * \\ Xosé Manuel Cid Fernández \\ María Victoria Carrera Fernández \\ Universidade de Vigo, España
}

\begin{abstract}
Los itinerarios académicos de exclusión escolar son mayoritarios entre el alumnado con expediente administrativo de protección. La declaración del estado de alarma, motivado por la alerta sanitaria del Covid-19, se traduce en un cambio en las dinámicas escolares. Esta investigación se propone analizar qué aspectos de la planificación docente, así como de la colaboración entre profesorado y profesionales de los recursos específicos de protección de Galicia, durante el estado de alarma, tienen incidencia en la inclusión o exclusión escolar del alumnado en dificultad social. Se realizan 11 seguimientos longitudinales con 11 adolescentes, cinco chicos y seis chicas. Asimismo, el estudio se diseña según la teoría fundamentada con la intención de triangular los discursos derivados de las entrevistas con el alumnado con estudios de casos instrumentales en los que participan 14 figuras profesionales. La muestra de equipos educativos está compuesta por 11 educadoras y tres educadores. Los resultados ponen de relieve que las dinámicas de desigualdad escolar se ven incrementadas durante el periodo del estado de alarma, observándose que el contacto entre alumnado y profesorado se reduce derivando en déficits en el trabajo de las tareas escolares; y que la colaboración entre figuras profesionales del sistema de protección y la escuela se limita a intercambios puntuales de información. Estos resultados destacan que, durante el estado de alarma motivado por la crisis sanitaria actual, la escuela y las políticas educativas en Galicia desatienden la situación de mayor dificultad social en la que se encuentra el alumnado en protección.
\end{abstract}

Descriptores: Educación inclusiva; Inclusión social; Infancia desfavorecida; Protección de la infancia; Estrategias educativas.

Students with administrative protection records are the protagonists of most of the academic itineraries of school exclusion. The declaration of the state of alarm, motivated by the health alert of Covid-19, leads to a change in the school dynamics of the students. This research aims to analyze which aspects of educational planning, as well as the collaboration between teachers and professionals of the specific protection resources of Galicia during the state of alarm, have an impact on the inclusion or exclusion of students in social difficulty. Eleven longitudinal follow-ups are carried out with 11 adolescents, five boys and six girls. The study is based on the grounded theory with the intention of triangulating the discourses derived from the interviews with students and instrumental case studies involving 14 professionals. The sample of educational teams consists of 11 female educators and 3 male educators. The results highlight that the dynamics of school inequality have increased during the period of the state of alarm, observing that the contact between students and teachers is reduced, which leads to deficits in the students' homework; and that the collaboration between the professionals in the protection system and the school is limited to timely exchanges of information. These results underline that, during the state of alarm motivated by the current health crisis, the school and education policies in Galicia neglect the situation of greater social difficulty of the students under protection.

Keywords: Inclusive education; Social inclusion; Disadvantaged childhood; Childhood protection; Educational strategies.

*Contacto: jesfernandez@uvigo.es

ISSN: 2254-3139

www.rinace.net/riejs/

revistas.uam.es/riejs
Recibido: $\quad 15$ de mayo 2020

$1^{\text {a }}$ Evaluación: 22 de junio 2020

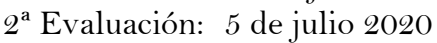

Aceptado: 14 de julio 2020 


\section{Introducción}

La infancia y la adolescencia en protección es aquella cuya situación de dificultad social es de tal magnitud que la administración pública se ve obligada a adoptar medidas para garantizar el respeto a su superior interés. Estamos ante un colectivo que presenta la mayor vulnerabilidad social de entre el alumnado que asiste a las escuelas. Asimismo, la ausencia de estadísticas oficiales sobre la evolución académica de la adolescencia con expediente de protección favorece la invisibilidad de este alumnado en el sistema educativo gallego. En este sentido, conocer la situación formativa del alumnado con medida administrativa protectora en Galicia hace imprescindible recurrir a las estadísticas de las entidades que trabajan con este colectivo. Los datos facilitados por el Programa mentor (IGAXES, 2018), configurados con una muestra de 3.680 participantes, indican que el 80\% de la juventud mayor de 16 años con expediente de protección activo en Galicia en el periodo 2010-2017 no dispone del Grado en Educación Secundaria Obligatoria (ESO). Los datos referidos al año 2017 indican que el 43,06\% ha cursado $3^{\circ}$ de ESO con retraso académico consolidado y que el $35,7 \%$ no ha superado $2^{\circ}$ de ESO. Teniendo en cuenta que repetir curso es un factor relacionado con el fracaso escolar (Bolívar y López, 2009), el panorama expuesto revela la situación de exclusión educativa que vivencia el colectivo.

Conocer la realidad de la juventud en dificultad social permite tomar consciencia de la importancia de desarrollar políticas activas para su inclusión escolar. Los sistemas de atención a la infancia y la escuela tienden a culpabilizarse mutuamente de la etiología de este problema (Fernández-Simo y Cid, 2016). La declaración del estado de alarma por la alerta sanitaria del Covid-19 sitúa a este grupo de alumnado en una situación de mayor vulnerabilidad en las escuelas, por lo que las estrategias socioeducativas que diseñe el sistema educativo minimizarán o incrementarán el impacto de la exclusión educativa durante este período excepcional. En esta línea, las experiencias que se desarrollen durante este período son una oportunidad de reflexión para incentivar el cambio hacia la mejora de la inclusión escolar.

\section{Revisión de la literatura}

Los itinerarios educativos de la adolescencia en protección han sido objeto de estudio de diversas investigaciones internacionales. Los resultados coinciden en indicar las graves dificultades de inclusión en el ámbito escolar (Barnow et al., 2015; Dixon, 2007; Montserrat et al., 2011; Okpych y Courtney, 2014). La precariedad de la intervención protectora y la etiología de las propias medidas de protección, derivan en dificultades específicas para la inclusión académica a las que no se les está dando una respuesta adecuada. Las consecuencias de esta exclusión educativa no solo son vivenciadas durante la etapa en protección. La repercusión durante la adultez se traduce en una perpetuación de la situación de vulnerabilidad social (Dregan y Gulliford, 2012; Vinnerljung y Hjern, 2011). La baja cualificación deriva en importantes dificultades para el acceso al mercado laboral (Dixon, 2007; Jurado, Olmos y Pérez, 2015; Okpych y Courtney, 2014).

El sistema de protección tiene como principal propósito que la adolescencia supere la situación de dificultad social que motivó la apertura del expediente de protección. El itinerario formativo, condiciona en gran medida que en la vida adulta se pueda romper la cadena de la exclusión social. La formación y el acceso al empleo se convierten en factores clave ante los déficits de apoyo familiar (Krinsky, 2010). La situación de vulnerabilidad de 
las familias impide que estas sean un apoyo efectivo para la superación de las metas propias de la transición a la vida adulta. La vida en dificultad social de la unidad de convivencia obstaculiza que pueda facilitar unas condiciones apropiadas para el desempeño académico. La prioridad de intervención de los recursos de protección se orienta a favorecer estrategias, para que los jóvenes hagan frente a posibles conflictos familiares (Cuenca, Campos y Goig, 2018). No quiere esto decir que se deba renunciar desde el sistema de protección a la intervención familiar. Al contrario, la investigación recopilada, indica que las prioridades de la acción profesional se orientan a dos dimensiones complementarias: Superar los déficits de intervención familiar presentes en el sistema (Melendro, De-Juanas y Rodríguez, 2017): Facilitar la autonomía de la juventud en la resolución propia de sus problemas vitales.

El término mayoría de edad, es para esta juventud sinónimo de obligación de contar con plena autonomía personal (Goyette, 2019). La administración no está obligada legalmente a dar soporte a las necesidades de la juventud ex tutelada una vez cumplidos los 18 años. Las reformas legislativas, presentadas en el año 2015, hacen referencia a la importancia de que las administraciones faciliten apoyo en el tránsito a la vida adulta mediante programas específicos. El marco legislativo no concreta la obligación de facilitar el acceso a estos recursos a toda la juventud con medida de protección. La política institucional sitúa a este colectivo en desigualdad respeto a sus coetáneos que dispongan de apoyo familiar (Bond, 2018). La baja cualificación y los déficits en capital social incrementan el riesgo de que esta juventud protagonice itinerarios vitales marcados por el desempleo y la precariedad (Stewart et al., 2014).

Un territorio que aspire a una escuela equitativa no puede permitirse que el alumnado con mayor dificultad social tenga altas probabilidades de terminar protagonizando un proceso de exclusión educativa. La equidad educativa requiere como condición, la igualdad de todo el estudiantado en el acceso a los aprendizajes de alta calidad sin obstáculos sociales (UNICEF, 2013). La escuela debe garantizar que la condición anterior se cumpla. A vista de lo expuesto, no solo no lo está haciendo, sino que está permitiendo una vulnerabilidad estructural que nos acerca a la importancia de asumir un proceso de cambio del sistema, orientado a la inclusión educativa (Fernández, 2017). La ausencia de políticas sociales eficaces se traduce en que el factor socioeconómico continúe muy presente en las motivaciones de los equipos técnicos del menor, justificativas de la apertura de expediente administrativo de protección (Fernández-Simo y Cid, 2020). La investigación viene relacionando el nivel socioeconómico y cultural del alumnado, con el logro educativo (Murillo y Román, 2011), siendo uno de los factores que incide en el itinerario educativo en el marco de una etiología multifactorial (Escudero, 2013; Murillo, 2008). La sociedad precisa de una escuela eficaz que alcance el desarrollo integral de todo el alumnado. Para este propósito, es imprescindible superar lo previsible en referencia a su contexto socioeconómico y cultural (Murillo, 2005). La meta es lograr un modelo de escuela proactiva en la definición de estrategias sistémicas para la inclusión escolar.

El propósito expuesto, requiere de un sistema educativo consciente de la importancia del trabajo colaborativo con otros actores sociales. La escuela no puede asumir en solitario la educación del alumnado, viéndose obligada a apelar a la responsabilidad de otros agentes e instituciones. El capital social de la escuela está condicionado por la red que teja con otras entidades, el cual crece si es utilizado, beneficiándose de la horizontalidad en las relaciones con el contexto (Bolívar, 2000). El trabajo educativo con la infancia y la adolescencia en protección exige de la colaboración entre sistemas. El uso del término 
"exige" no es casual, ya que sin dinámicas colaborativas no se alcanzará el objetivo compartido. La colaboración no debería limitarse a aspectos de carácter burocrático, sino que se señalan como importantes la relaciones entre figuras profesionales (FernándezSimo y Cid, 2016). Los escenarios de encuentro e intercambio son parte del proceso en la definición de una estrategia compartida. La perspectiva de escuela inclusiva lleva inherente la presencia de procesos que faciliten la participación de todo el alumnado en la comunidad educativa, en los contenidos y en la cultura escolar, especialmente de aquellos estudiantes que presentan mayor riesgo de exclusión (Booth y Ainscow, 2002).

La colaboración con otros agentes nos aproxima a una escuela abierta y consciente de la importancia de superar barreras autoimpuestas. El análisis y la identificación de las dimensiones del sistema educativo que favorecen la inequidad requiere de un enfoque ecológico-sistémico (Sicilia y Simancas, 2018). La realidad integral del alumnado forma parte de un correcto diagnóstico socioeducativo. El factor social tiene que estar presente en el diseño y en la evaluación de las estrategias escolares. El contexto incide en la eficacia de la actuación docente, haciendo inviable una planificación ajena a la realidad social del alumnado. Las dinámicas de relación, entre escuela y equipos profesionales del sistema de protección, incidirán en la coherencia del mensaje educativo que se traslade a la adolescencia en dificultad social. La tirantez o la flexibilidad con la que se diseñe o se afronte esa relación será vivenciada por todos los actores implicados, incluido el alumnado. La interacción con otros agentes educativos, en este caso los recursos de protección, lleva implícito el reconocimiento del papel que estos equipos pueden tener en la facilitación de la inclusión escolar, objetivo final compartido por ambos sistemas.

Coincidiendo con la declaración del estado de alarma, el equipo de investigación se encuentra realizando un estudio, mediante seguimientos longitudinales, analizando los itinerarios formativos y laborales de la juventud con medida administrativa de protección y su incidencia en el proceso de transición a la vida adulta. La declaración del estado de alarma origina que se detecten nuevas dificultades para continuar con la actividad escolar. El confinamiento deriva en una oportunidad para observar, desde fuera de la escuela, las estrategias utilizadas por el profesorado. La actividad docente queda expuesta a la mirada de los equipos profesionales de los recursos de protección. Las educadoras y educadores, que habitualmente acompañan a este alumnado desde fuera de la escuela, asumen durante este periodo una labor extra en la supervisión de la realización de la tarea escolar. La complejidad propia de los procesos pedagógicos puede analizarse desde diversidad de perspectivas culturales y subjetivas. La información facilitada por alumnado y profesionales de la acción socioeducativa con adolescencia en dificultad social representa la vivencia en primera persona de como las cuestiones relacionadas con el factor social afectan al itinerario académico. La situación excepcional expuesta se convierte en un escenario privilegiado para visibilizar cómo opera la escuela en relación con el alumnado en protección en Galicia. Los objetivos de la investigación son: 1) Analizar qué aspectos de la planificación docente, tienen incidencia en la inclusión o exclusión escolar del alumnado en dificultad social durante el estado de alarma; y 2) Valorar las dinámicas establecidas de colaboración entre profesorado y profesionales de los recursos específicos de protección fruto de la alerta sanitaria del Covid-19. 


\section{Método}

La metodología que se diseña es resultado de una oportunidad improvista que surge durante un estudio más amplio. La investigación trata de convertirse en una oportunidad de pensar la inclusión y la exclusión superando barreras disciplinares (Susinos y Parrilla, 2013). Se detectan desde el sistema de protección aspectos de interés derivados de la actuación de la escuela. Durante los seguimientos longitudinales es la propia voz de la juventud el medio para la profundización de la situación (Parrilla, Gallego y Morriña, 2010). Se utiliza una muestra intencional compuesta por 11 jóvenes con una edad media de 18,3 años (cuadro 1). Se informa telefónicamente a la juventud participante de la finalidad del estudio, de la confidencialidad de la información que se obtiene y se solicita su consentimiento verbal. Los seguimientos longitudinales se realizan durante 23 días, iniciándose el día 18 de marzo del 2020 y finalizando el 9 de abril del 2020. El número total de contactos es 42 , de los que 25 son telefónicos y 14 son video llamadas por WhatsApp. La media de contactos por joven es de 3,8. La juventud participante reside en la provincia de Ourense. Durante los seguimientos el interés de la investigación se centra en las siguientes interrogantes: Disposición del alumnado de recursos adecuados (tablets, wifi, pc..) para la continuidad de la actividad académica; Contactos del profesorado con el alumnado; Supervisión docente de las actividades escolares; Atención a las dificultades sociales concretas de cada caso y adaptabilidad a las circunstancias; Colaboración y relación de profesorado y figuras profesionales del sistema de protección, según la visión del alumnado.

Cuadro 1. Muestra de jóvenes participantes en los seguimientos longitudinales

\begin{tabular}{|c|c|c|c|c|c|}
\hline \multirow{2}{*}{ Código } & \multirow{2}{*}{ EsTUDIOS } & \multirow{2}{*}{ GÉNERO } & \multirow{2}{*}{ EDAD } & \multicolumn{2}{|c|}{ CONTACTOS } \\
\hline & & & & Telefónicos & Videollamada \\
\hline SL1 & $1^{\circ} \mathrm{GM}$ & Femenino & 18 & 2 & 1 \\
\hline $\mathrm{SL} 2$ & $2^{\circ} \mathrm{FPB}$ & Femenino & 20 & 3 & 1 \\
\hline SL3 & $2^{\circ} \mathrm{FPB}$ & Masculino & 18 & 2 & 1 \\
\hline SL4 & $2^{\circ} \mathrm{FPB}$ & Masculino & 18 & 5 & $\mathrm{O}$ \\
\hline SL5 & $3^{\circ} \mathrm{ESO}$ & Femenino & 18 & 4 & $\mathrm{O}$ \\
\hline SL6 & $2^{\circ} \mathrm{BACH}$ & Masculino & 18 & 2 & 1 \\
\hline SL7 & $2^{\circ} \mathrm{GM}$ & Femenino & 19 & 1 & 3 \\
\hline SL8 & $3^{\circ} \mathrm{ESO}$ & Masculino & 18 & 1 & 2 \\
\hline SL9 & $2^{\circ} \mathrm{FPB}$ & Femenino & 18 & 3 & $\mathrm{O}$ \\
\hline SL10 & $4^{\circ} \mathrm{ESO}$ & Femenino & 18 & 1 & 2 \\
\hline SL1 1 & $4^{\circ} \mathrm{ESO}$ & Masculino & 18 & 1 & 3 \\
\hline
\end{tabular}

Nota: Grado Medio (GM); Grado Superior (GS); Formación Profesional Básica (FPB); Bachillerato (BACH).

Fuente: Elaboración propia.

La información obtenida en los seguimientos longitudinales, con la juventud en protección, se complementa con la facilitada por figuras profesionales mediante el análisis de estudio de casos instrumentales. La metodología permite contar con la perspectiva de los actores implicados en la realidad educativa del sistema de protección. Se realizan entrevistas con educadoras y educadores de equipos educativos de recursos de intervención social en las que se analiza una diversidad de perspectivas. El proceso facilita la comprensión de un entorno humano determinado (Stake, 2005), como es el caso del sistema de protección. Se utiliza una metodología propia de la teoría fundamentada. La 
información de los seguimientos longitudinales es la base para la realización de entrevistas semiestructuradas con miembros de los equipos educativos. En las entrevistas con figuras profesionales se procuró atender a las siguientes interrogantes: Facilitación de medios para poder realizar las actividades vía virtual; Contactos del profesorado con el alumnado interesándose por su situación; Explicaciones previas de las tareas escolares; Trabajo posterior de las actividades realizadas; Planificación de los deberes escolares en función las circunstancias del alumnado en dificultad social; Trabajo colaborativo del profesorado con profesionales del sistema de protección.

Cuadro 2. Muestra profesionales participantes en el estudio de casos

\begin{tabular}{|c|c|c|c|c|c|c|c|}
\hline $\begin{array}{c}\text { TIPOLOGÍA } \\
\text { RECURSO }\end{array}$ & CódIGo & & JDIANTES & EDAD & GÉNERO & $\begin{array}{c}\text { MESES DE } \\
\text { EXPERIENCIA } \\
\text { LABORAL }\end{array}$ & Provincia \\
\hline \multirow{19}{*}{ Residencial } & & 1 & FPB & & & & \multirow{7}{*}{ Pontevedra } \\
\hline & $\mathrm{E} 1$ & 2 & SEC & 28 & Femenino & 31 & \\
\hline & & 1 & GM & & & & \\
\hline & \multirow{3}{*}{$\mathrm{E} 2$} & 1 & $\mathrm{BACH}$ & \multirow{3}{*}{35} & \multirow{3}{*}{ Femenino } & \multirow{3}{*}{63} & \\
\hline & & 1 & SEC & & & & \\
\hline & & 2 & FPB & & & & \\
\hline & E3 & 3 & SEC & 32 & Masculino & 49 & \\
\hline & \multirow{2}{*}{$\mathrm{E} 4$} & 1 & GM & \multirow{2}{*}{29} & \multirow{2}{*}{ Femenino } & \multirow{2}{*}{86} & \multirow{12}{*}{ Ourense } \\
\hline & & 1 & $\mathrm{FPB}$ & & & & \\
\hline & E5 & 3 & SEC & 34 & Femenino & 101 & \\
\hline & \multirow{2}{*}{ E6 } & 2 & FPB & \multirow{2}{*}{42} & \multirow{2}{*}{ Masculino } & \multirow{2}{*}{142} & \\
\hline & & 1 & SEC & & & & \\
\hline & \multirow{2}{*}{ E7 } & 3 & FPB & \multirow{2}{*}{46} & \multirow{2}{*}{ Femenino } & \multirow{2}{*}{165} & \\
\hline & & 1 & GM & & & & \\
\hline & \multirow{3}{*}{ E8 } & 3 & FPB & \multirow{3}{*}{41} & \multirow{3}{*}{ Femenino } & \multirow{3}{*}{57} & \\
\hline & & 1 & $\mathrm{BACH}$ & & & & \\
\hline & & 2 & GM & & & & \\
\hline & \multirow{2}{*}{ E9 } & 4 & SEC & \multirow{2}{*}{40} & \multirow{2}{*}{ Femenino } & \multirow{2}{*}{42} & \\
\hline & & 2 & $\mathrm{FPB}$ & & & & \\
\hline \multirow{14}{*}{ Comunitario } & E10 & 3 & FPB & 25 & Femenino & 15 & \\
\hline & & 1 & SEC & 25 & & & Pontevedra \\
\hline & & 1 & GM & & & & Ponteveara \\
\hline & E11 & 4 & $\mathrm{FPB}$ & 31 & Femenino & 21 & \\
\hline & & 1 & GS & & & & \\
\hline & E12 & 1 & $\mathrm{BACH}$ & 39 & & 72 & \\
\hline & L12 & 3 & GM & 39 & Mrascumo & & \\
\hline & & 4 & SEC & & & & \\
\hline & & 5 & FPB & & & & \\
\hline & E13 & 6 & SEC & 33 & Femenino & 95 & Ourense \\
\hline & & 3 & GM & & & & \\
\hline & & 1 & GM & & & & \\
\hline & E 14 & 5 & SEC & 39 & Femenino & 26 & \\
\hline & & 3 & FPB & & & & \\
\hline
\end{tabular}

Notas: Secundaria (SEC); Grado Superior (GS).

Fuente: Elaboración propia.

Los casos se estudian mediante una muestra configurada por conveniencia con 14 profesionales que llevan en activo en el sistema de protección un mínimo de 12 meses (cuadro 2). Según la tipología de recurso en la que trabajan, nueve profesionales realizan su intervención en unidades residenciales y otras cinco figuras profesionales se encargan del acompañamiento socioeducativo en recursos comunitarios, es decir, en equipos que realizan la intervención en programas de acompañamiento socioeducativo con menores 
con expediente de protección y que conviven con su familia. Nueve participantes trabajan en la provincia de Ourense y cinco en la de Pontevedra. Las educadoras y educadores acompañan, durante el estado de alarma, a un total de 76 estudiantes que cursan los siguientes estudios: 29 de formación profesional básica; 30 de secundaria; 13 de grado medio; tres de bachillerato; uno grado superior. La edad media de las figuras profesionales es de 35,3 años. El tiempo medio de experiencia laboral especializada en meses es de 68,9. Las entrevistas se realizan con la aplicación informática Skype entre el 13 y el 24 de abril del año 2020. Previamente se mantiene un contacto telefónico en el que se solicita la colaboración, informando sobre las cuestiones de interés para la investigación y los datos que son necesarios.

El análisis de datos se realiza con la colaboración de dos figuras profesionales externas al equipo de investigación. Ambos profesionales cuentan con dilatada experiencia en el sistema de protección y formación especializada. Disponen de un conocimiento profundo de la realidad de las cuestiones a investigar. Esta colaboración externa da confiabilidad a la investigación. Los resultados se analizan estudiando la frecuencia en la muestra.

\section{Resultados}

Los resultados obtenidos en los seguimientos longitudinales y en los estudios de caso se presentan simultáneamente. Se agrupan en función de la categoría de análisis. La categorización de los discursos de alumnado y profesionales facilita la estructuración de la presentación de los resultados. La intención es que el texto sirva de refuerzo de la información resumida en el cuadro 3. A modo de ejemplo, se presentan argumentos recogidos durante el proceso de investigación. La voz de alumnado y profesionales participantes representa la viveza del hecho pedagógico en primera persona.

El contacto del profesorado con el alumnado durante el estado de alarma varía en función del docente. Los resultados indican que, en el mismo centro educativo, hay docentes que contactan telefónicamente una vez por semana y otros casos en los que el contacto se limita a un mail con indicaciones sobre las actividades a realizar. "La relación con los chicos depende directamente del profesor que sea. Ves profesores muy implicados que están muy pendientes y otros que se limitan a enviar indicaciones" (E11), comenta una educadora. Una joven estudiante de formación profesional básica manifiesta que "la ayuda de la tutora está siendo muy grande resolviendo por teléfono las dudas” (SL2). Otra alumna que cursa los mismos estudios en un centro educativo diferente argumenta lo contrario. "Nadie se pone en contacto conmigo y cuando llamo solo doy hablado con la directora que me dice que haga lo que me envían por mail” (SL9), dice la joven.

La situación es diversa en relación a la facilitación de medios virtuales para el seguimiento de la actividad académica. "La profesora me llamó y me preguntó si tenía problemas con la conexión a internet. Me dijo que si lo necesitaba me ofrecía una ayuda” (SL6), comenta un joven que estudia bachillerato. Otro estudiante, en este caso de secundaria, afirma que "los profesores te suben lo que tienes que hacer, pero no te dicen que hacemos los que no tenemos portátil en casa” (SL11). Una educadora asegura que la ayuda efectiva para la superación de las dificultades de medios está concentrada en entidades del tercer sector. "Me enfada mucho que la Consellería de Educación, haga anuncios en prensa de que nadie se va a quedar atrás, pero después en la práctica todo queda en nada, se centran los apoyos en alguna ONG que facilita las cosas" (E13), dice la profesional. 
Cuadro 3. Planificación docente y colaboración profesional en la acción educativa con alumnado en protección durante el estado de alarma

\begin{tabular}{|c|c|c|c|c|}
\hline & & \multicolumn{2}{|c|}{ FRECUENCIAS } & \multirow{2}{*}{$\begin{array}{c}\text { FrEC. } \\
\text { ABSOLUTA }\end{array}$} \\
\hline & & Seguimientos & Estudio de casos & \\
\hline \multicolumn{5}{|c|}{ Planificación docente } \\
\hline \multirow{5}{*}{$\begin{array}{l}\text { Incidendia } \\
\text { inclusión escolar }\end{array}$} & Contacto semanal telefónico entre profesorado y alumnado & $\begin{array}{l}\text { SL2, SL3, SL4, SL6 } \\
\end{array}$ & E1, E3, E5, E7, E10, E11 & 10 \\
\hline & Facilitación de medios para la realización de actividades escolares & SL1, SL2, SL3, SL4, SL6, SL10 & $\begin{array}{l}\text { E1, E3, E7, E8, E9, E10, E11, } \\
\text { E,14 }\end{array}$ & 14 \\
\hline & Explicación previa de las tareas escolares por medios virtuales & SL2, SL3, SL4, SL6, SL10 & E1, E3, E11, E14 & 9 \\
\hline & $\begin{array}{l}\text { Trabajo educativo posterior de las tareas escolares mediante } \\
\text { seguimientos virtuales }\end{array}$ & SL2, SL3, SL4, SL6 & E1, E3, E9, E11 & 8 \\
\hline & $\begin{array}{l}\text { Adaptabilidad y flexibilidad de las tareas en función de la } \\
\text { situación social }\end{array}$ & SL3, SL6 & E1, E3, E11 & 5 \\
\hline \multirow{5}{*}{$\begin{array}{l}\text { Incidencia } \\
\text { exclusión } \\
\text { escolar }\end{array}$} & $\begin{array}{l}\text { Ausencia de contacto semanal telefónico entre profesorado y } \\
\text { alumnado }\end{array}$ & $\begin{array}{l}\text { SL1, SL5, SL7, SL8, SL9, SL10, } \\
\text { SL11 }\end{array}$ & $\begin{array}{l}\text { E2, E4, E6, E8, E9, E12, E13, } \\
\text { E14. }\end{array}$ & 15 \\
\hline & $\begin{array}{l}\text { No puesta en contacto para interesarse sobre la necesidad de } \\
\text { facilitación de medios para el seguimiento de la actividad escolar }\end{array}$ & SL5, SL7, SL8, SL1 1 & $\mathrm{E} 2, \mathrm{E} 4, \mathrm{E} 5, \mathrm{E} 6, \mathrm{E} 12, \mathrm{E} 13$ & 10 \\
\hline & Tareas escolares sin explicación previa & SL1, SL5, SL7, SL8, SL9, SL1 1 & $\begin{array}{l}\text { E2, E4, E5, E6, E7, E8, E9, } \\
\text { E10, E12, E13 }\end{array}$ & 16 \\
\hline & No hay trabajo educativo posterior de las tareas escolares & $\begin{array}{l}\text { SL1, SL5, SL7, SL8, SL9, SL10, } \\
\text { SL1 } 1\end{array}$ & $\begin{array}{l}\text { E2, E4, E5, E6, E7, E8, E10, } \\
\text { E12, E13, E14 }\end{array}$ & 17 \\
\hline & $\begin{array}{l}\text { Sin adaptación de las tareas escolares en función de las } \\
\text { circunstancias sociales }\end{array}$ & $\begin{array}{l}\text { SL1, SL2, SL4, SL5, SL7, SL8, } \\
\text { SL9, SL10, SL11 }\end{array}$ & $\begin{array}{l}\text { E2, E4, E5, E6, E7, E8, E9, } \\
\text { E10, E12, E13, E14 }\end{array}$ & 20 \\
\hline \multicolumn{5}{|c|}{ Colaboración entre profesorado y equipos educativos de protección } \\
\hline \multirow{3}{*}{$\begin{array}{l}\text { Dinámicas } \\
\text { facilitadoras }\end{array}$} & $\begin{array}{l}\text { Contactos frecuentes previos al estado de alarma entre figuras } \\
\text { profesionales }\end{array}$ & SL3, SL4, SL6, SL10 & $\mathrm{E} 1, \mathrm{E} 3, \mathrm{E} 7, \mathrm{E} 10, \mathrm{E} 11$ & 9 \\
\hline & Contactos entre profesionales durante el estado de alarma & $\begin{array}{l}\text { SL1, SL2, SL3, SL4, SL6, SL7, } \\
\text { SL10 }\end{array}$ & E1, E3, E5 E7, E10, E11, E13 & 14 \\
\hline & Colaboración en el diseño de estrategias educativas & SL6 & E11 & 2 \\
\hline \multirow{3}{*}{$\begin{array}{l}\text { Dinámicas } \\
\text { obstaculizadoras }\end{array}$} & Contactos ocasionales previos al estado de alarma & SL1, SL2, SL5, SL7, SL8, SL9 & $\mathrm{E} 2, \mathrm{E} 4, \mathrm{E} 8, \mathrm{E} 12$ & 10 \\
\hline & Sin contactos durante estado de alarma & SL5, SL8, SL9, SL1 1 & $\mathrm{E} 2, \mathrm{E} 4, \mathrm{E} 6, \mathrm{E} 8, \mathrm{E} 9, \mathrm{E} 12, \mathrm{E} 14$ & 11 \\
\hline & $\begin{array}{l}\text { Sin diseño compartido entre profesionales de ambos sistemas de } \\
\text { las estrategias educativas }\end{array}$ & $\begin{array}{l}\text { SL1, SL2, SL3, SL4, SL5, SL7, } \\
\text { SL8, SL9, SL10, SL11 }\end{array}$ & $\begin{array}{l}\text { E1, E2, E3, E4, E5, E6, E7, } \\
\text { E8, E9, E10, E12, E13, E14 }\end{array}$ & 22 \\
\hline
\end{tabular}

Fuente: Elaboración propia. 
El trabajo educativo de las tareas escolares no presenta un patrón homogéneo. Se detectan situaciones variables. Los resultados indican que el trabajo educativo de los ejercicios que se realizan en casa depende de cada docente. "La mayoría de las profesoras hacen una explicación previa en un video o en la plataforma" (E1), dice una profesional. "Los profes nos cuelgan las explicaciones en la página y luego realizamos los ejercicios" (SL10), dice una joven que estudia ESO. "Hacemos lo que nos suben, pero no nos los explican" (SL8), comenta otro estudiante. El trabajo educativo posterior de los ejercicios escolares no se realiza en todos los casos. "Lo que veo es que se ponen unos deberes y luego comunican si están bien o no, pero no se trabaja con ellos" (E7), afirma una profesional. Otra educadora comenta que "habitualmente ve como se hace un seguimiento desde el instituto de las tareas escolares y se explican las cosas que no quedaron claras" (E9).

Los resultados indican que son escasas las situaciones en las que se lleva a cabo una adaptabilidad de la acción educativa al contexto social de alumnado. Una de estas excepciones detectadas, es la de un joven que estudia formación profesional básica. "La profesora me ayudó a conseguir datos para el teléfono y así poder ver lo que sube... Cuando no se me descargan las cosas ella me llama y las hacemos por teléfono" (SL3), comenta el alumno. Un educador manifiesta que "hay profesores que si son flexibles y tratan de adaptarse a la realidad del alumnado" (E3). En los seguimientos longitudinales se detecta una ausencia de adaptación de la realidad social del alumnado. "Le dije al de historia que no tenía portátil y me dijo que en una casa hoy en día tiene que haber un ordenador igual que hay móviles" (SL8), manifiesta un estudiante de ESO. "En los centros vamos arreglándonos con los medios que tenemos, pero los que se fueron para casa tienen la situación más complicada” (E5), comenta un profesional. Precisamente una educadora, que realiza su trabajo en medio abierto, afirma que "la tutora de una chica me dijo literalmente que ella no podía hacer más con un profesor que pedía que se le mandase todo por ordenador y no atendía el teléfono... no entiendo cómo es posible que se permitan situaciones así, sabiendo que el chico no tiene ordenador en casa" (E14).

Los resultados analizados indican que, en la mayoría de las situaciones, las dinámicas de colaboración, entre profesorado y equipos educativos del sistema de protección, se limitan a contactos puntuales de intercambio de información. En algunos casos hay relación previa a la declaración del estado de alarma. "Mi educadora habla de vez en cuando con mi tutora y así ya saben en el centro como voy en las clases" (SL10), comenta una estudiante de ESO. "Ya antes de todo esto hablaba puntualmente con las profesoras de los chicos" (E7), dice una profesional. Los contactos durante la alerta sanitaria varían según la situación analizada. "Durante todo este tiempo no tengo manera de hablar por teléfono con la mayoría de los profes... solo consigo hacerlo por mail" (E2), afirma una educadora. Los seguimientos indican que son frecuentes las dificultades para establecer un canal fluido de comunicación con el profesorado. "Le dije a mi educador que hablase con las profes para ver como hacíamos... solo hablan por mail y en los deberes hay cosas que no damos entendido" (SL9), dice una estudiante de formación profesional básica.

Los casos analizados, indican que la colaboración en el diseño de la acción educativa, entre profesionales de ambos sistemas, se da en situaciones muy puntales. Es el caso expuesto por una educadora que se alegra de "la buena disposición para acordar siempre como hacemos cuando alguno de los chicos tiene algún problema” (E11). Únicamente esta profesional y uno de los seguimientos longitudinales aportan información sobre estrategias de colaboración conjunta entre escuela y equipos educativos de protección. Los casos apuntan a estrategias educativas no diseñadas en conjunto. "Sería bueno que todas 
remásemos en la misma dirección, pero para eso tenemos que hablar más y coordinarnos, no solo hablar cuando tienen un problema con un chico" (E8), dice una profesional. Varios argumentos indican que cuando la demanda de contacto proviene de la escuela suele ser por conflictos con el alumnado (E6, E12). Otra educadora afirma que "es frecuente que desde la escuela no se conozca con claridad la realidad de los chicos" (E9). "Los equipos educativos de uno y otro lado tenemos información de interés para el otro, pero no debería ser solo un intercambio de información sino una actuación coordinada" (E14), argumenta una educadora, asegurando que esta colaboración es aún más decisiva en momentos tan excepcionales como la alerta sanitaria.

\section{Discusión y conclusiones}

El presente artículo analiza las estrategias utilizadas por la escuela durante el estado de alarma con el alumnado en protección. Los resultados indican que las actuaciones docentes son ajenas a la dificultad social. En este sentido, superan su intencionalidad como instrumento con el que conseguir una finalidad educativa. Las estrategias tienen un significado que nos aproxima a la comprensión que los actores tienen de la realidad social del alumnado. Siguiendo a Escudero (2012), las estrategias tienen su importancia en la inclusión educativa, pero son de mayor relevancia la cultura y la política educativa como motores para favorecer la implicación colectiva. El estudio indica diversidad de estrategias de los centros educativos. La riqueza de formas de afrontar la situación, algunas de ellas contradictorias entre sí, puede ser resultado de una ausencia de política institucional del sistema educativo gallego, especialmente en el papel de la escuela como agente activo de inclusión social.

Los resultados indican déficits en la estrategia socioeducativa de la escuela, lo que apunta a una falta de cultura institucional acerca de la importancia del factor social en el marco escolar. La respuesta a las necesidades del colectivo no se ejecuta mediante una acción puntual, sino con un cambio de la cultura de la institución (Murillo y Krichesky, 2012). La adolescencia en protección que alcanza sus objetivos escolares es una minoría. En los casos en los que, si se produce el logro de las metas académicas, todo indica que, tal y como señalan Susinos y Parrilla (2013), se debe principalmente a la capacidad individual de las personas para superar condiciones adversas en el proceso de inclusión. Los resultados apuntan a déficits del sistema educativo en el apoyo en los procesos académicos del colectivo.

El alumnado en protección no dispone de un acompañamiento práctico por parte de la escuela durante el estado de alarma que le permita superar la situación de dificultad social en la que se encuentran. La relación entre profesorado y alumnado se centra en la reproducción de contenidos curriculares previamente presentados por el docente (Hernández, Murillo y Martínez-Garrido, 2014), permaneciendo ajena la situación social que rodea la acción educativa. Los resultados indican, que incluso en algunos casos no se produce trabajo educativo de las tareas escolares. En situaciones como la expuesta, los deberes no cumplen con su función como herramienta educativa orientada a la mejora de los resultados académicos. El profesorado que realiza un trabajo individualizado de la tarea escolar consigue incrementar el rendimiento escolar y mejorar la satisfacción del alumnado con la escuela (Martínez-Garrido y Murillo, 2016). Los resultados indican una variedad en los deberes escolares que, coincidiendo con Protheroe (2009), responden a preferencias docentes y son planificados sin contar con la realidad del alumnado. 
Favorecer la participación activa del estudiantado en protección en la vida escolar, podría ser un elemento facilitador de la inclusión. Investigaciones previas, desarrolladas en el sistema de protección, indican que la participación y la claridad del proyecto socioeducativo favorecen la implicación en la consecución de las metas personales (Comasólivas, Sala-Roca y Marzo, 2018; Cassarino-Perez, Córdova, Montserrat, Sarriera, 2018), destacando la interacción y la participación como elementos imprescindibles para la buena atención institucional (Jussila, 2016). A modo de ejemplo de lo expuesto, retomando la cuestión de los deberes, los resultados de la investigación indican, que el alumnado en protección verbaliza discrepancias sobre la utilidad de las tareas escolares. Al mismo tiempo, las figuras profesionales, participantes en el presente estudio, manifiestan que no observan que los deberes realizados tengan refuerzo posterior por parte del cuerpo docente. La situación expuesta se traduce en que la tarea escolar pierda su efecto positivo en el rendimiento y en el autoconcepto del alumnado, al no ser utilizada posteriormente por el profesorado (Murillo y Martínez-Garrido, 2014).

Las dinámicas de relación entre docentes y alumnado están condicionadas por la situación del estado de alarma. Las carencias de medios (portátiles o tablets), así como las deficiencias en conexiones a internet, sitúan a este colectivo en un contexto de mayor vulnerabilidad. En estas condiciones parece crucial la empatía del docente, como cualidad humana facilitadora de una relación eficaz y profunda (Fernández-Salinero, 2014). Los resultados indican que las estrategias de superación de las dificultades dependen en exclusiva de la predisposición docente. Una investigación realizada en Galicia con 6.350 docentes concluye que el $61 \%$ del profesorado considera que la comunicación con el alumnado se realizó con dificultades, siendo el $82 \%$ de esas limitaciones derivadas de la situación social (Bermello, 2020). Los resultados del presente trabajo coinciden en que la brecha digital tiene especial incidencia en el alumnado que vive en unidades de convivencia en dificultad social (Save de Children, 2020); sin embargo, no indican déficits de medios tecnológicos en los y las estudiantes que permanece en recursos residenciales. El sistema educativo como institución permanece ajeno a la realidad analizada. En las situaciones de dificultad social cobran aún más peso las interacciones entre alumnado y docentes. El profesorado implicado estructura las relaciones en función de dimensiones éticas, morales y afectivas (Orellana-Fernández, Merellano-Navarro y Almonacid-Fierro, 2018). La implicación ética de la educación equitativa se refleja en el compromiso con la cohesión social y en la apuesta colectiva por una ciudadanía digna (Simón, Barrios, Gutiérrez y Muñoz, 2020). En el marco de la expuesta dejación de funciones de la administración, el peso de la acción educativa efectiva recae en las figuras profesionales. Durante el estado de alarma, se acentúan las consecuencias de la omisión de responsabilidades institucionales por parte del sistema educativo. El clima de atención y apoyo en la respuesta que se da a la situación de dificultad social queda en manos de centros educativos y del cuerpo docente (Escudero, 2012).

Las figuras profesionales externas al sistema educativo, que acompañan a juventud en dificultad social, consideran pendiente e imprescindible la colaboración entre los equipos de los recursos sociales y el cuerpo docente. Las educadoras y educadores de protección manifiestan que la predisposición inicial del profesorado se sitúa en colaboraciones puntuales, limitadas a un intercambio de información. Argumentan que habitualmente la iniciativa de contacto suele ser de los equipos de intervención socioeducativa. Apuntan a que las escuelas son proactivas en la demanda de colaboración cuando tienen algún conflicto con el alumnado. Investigaciones previas señalan que el profesorado no considera 
una prioridad la colaboración con otros actores sociales (Fernández-Larragueta, Fernández-Sierra y Rodorigo ,2014). La escuela tendrá mayores dificultades en hacer frente en solitario a las necesidades detectadas. La innovación docente necesita del trabajo en entornos colaborativos en los que se compartan las responsabilidades y se fomente la reflexión (Krichesky y Murillo, 2018).

La propuesta de educación inclusiva de la administración gallega se centra en la atención a necesidades concretas de alumnado con diversidad funcional. La investigación indica que la escuela en Galicia permanece ajena al estudiantado con medida administrativa de protección. Se puede concluir que no estamos ante una escuela inclusiva, ya que para referirse en estos términos tendríamos que hablar de una escuela que tenga en cuenta las necesidades de todo el alumnado (Miles y Singal 2010), dando respuesta a situaciones socialmente diversas y a expectativas variadas (Escudero y Martínez, 2011). La escuela en Galicia tiene pendiente adoptar una práctica sistemática proactiva en la facilitación de la inclusión escolar del alumnado en protección.

\section{Referencias}

Barnow, B. S., Buck, A., O'Brien, K., Pecora, P., Ellis, M. L. y Steiner, E. (2015). Effective services for improving education and employment outcomes for children and alumni of foster care service: Correlates and educational and employment outcomes. Child ङंFamily Social Work, 2O(2), 159-170. https://doi.org/10.1111/cfs.12063

Bermello, S. (2020). Retrato da teledocencia forzada: Elevada desconexión, posta en valor da educación presencial e suspenso xeral da consellería. Revista Galega de Educación, 1, 24-27.

Bolívar, A. (2006). Familia y escuela: Dos mundos llamados a trabajar en común. Revista de Educación, 339, 119-146.

Bolívar, A. y López, L. (2009). Las grandes cifras del fracaso escolar y los riesgos de exclusión educativa. Profesorado, Revista de Curriculum y Formación del Profesorado, 13(3), 51-78.

Bond, S. (2018). Care-leaving in South Africa: An international and social justice perspective. Journal of International and Comparative Social Policy, 34(1), 76-90. https://doi.org/10.1080/21699763.2017.1413994

Cassarino-Perez, L., Córdova, V. E., Montserrat, C. y Sarriera, J. C. (2018). Transição entre o acolhimento e a vida adulta: Uma revisão sistemática sobre intervenções. Trends in Psychology, 26(3), 1665-1681. https://doi.org/10.9788/TP2018.3-19

Comasólivas, A., Sala-Roca, J. y Marzo, T. E. (2018). Los recursos residenciales para la transición a la vida adulta de los jóvenes tutelados. SIPS-Pedagogía Social. Revista Interuniversitaria, 31 , 125-137. https://doi.org/10.7179/PSRI_2018.31.10

Cuenca, M. E., Campos, G. y Goig, R. M. (2018). El tránsito a la vida adulta de los jóvenes en acogimiento residencial: El rol de la familia. Educación XX1, 21(1), 321-344. https://doi.org/10.5944/educXX1.16510

Dixon, J. (2007). Obstacles to participation in education, employment and training for young people leaving care. Social Work and Social Sciences Review, 13(2), 18-34. https://doi.org/10.1921/19648

Dregan, A. y Gulliford, M. C. (2012). Foster care, residential care and public care placement patterns are associated with adult life trajectories: population based cohort study. Social Psychiatry and Psychiatric Epidemiology, 47(9), 1517-1526. https://doi.org/10.1007/s00127-011-0458-5 
Escudero, J. M. (2012). La educación inclusiva, una cuestión de derecho. Educatio Siglo XXI, 30(2), 109-128.

Escudero, J. M. (2013). Estudiantes en riesgo, centros escolares en riesgo: Respuestas educativas al alumnado en situación de vulnerabilidad. DM Editor.

Escudero, J. M. y Martínez, B. (2012). Las políticas de lucha contra el fracaso escolar: ¿Programas especiales o cambios profundos del sistema y la educación? Revista de Educación, número extraordinario, 174-193. https://doi.org/10.4438/1988-592X-RE-2012-EXT-2 11

Fernández, J. (2017). Alumnado inmigrante en la ESO: Vulnerabilidad pedagógica del sistema educativo. Educación XXI, 2O(1), 12 1-140. https://doi.org/10.5944/eduXXI.12855

Fernández-Larragueta, S., Fernández-Sierra, J. y Rodorigo, M. (2014). Coordinación interprofesional en los centros educativos: Una apuesta para la inclusión. Estudios sobre Educación, 27, 193-211. https://doi.org/10.15581/004.27.193-211

Fernández-Salinero, C. (2014). La tutoría universitaria en el escenario europeo de educación superior: Perfiles actuales. Teoría de la Educación, 26(1), 161-186. https://doi.org/10.14201/teoredu2014261161186

Fernández-Simo, D. y Cid, X. M. (2020). Adolescencias invisibles: A educación social nos camiños do empoderamento. Andavira.

Fernández-Simo, D. y Cid, X. M. (2016). The underage protection system in Galicia (Spain). New needs for social and educational support with teens and youth in social difficulties. En T. Suikkanen-Malin, M. Vestila y J. Jussila (Eds.), Foster Care, Childhood and \& Parenting in Contemporary Europe (pp. 31-44). Publications of Kymenlaakso University of Applied Sciences.

Forsman, H., Brännström, L., Vinnerljung, B. y Hjern, A. (2016). Does poor school performance cause later psychosocial problems among children in foster care? Evidence from national longitudinal registry data. Child Abuse \& Neglect, 57, 61-71.

https://doi.org/10.1016/j.chiabu.2016.06.006

Goyette, M. (2019). Social networks and social support in the transition to adulthood: A reaction on social integration processes. En V. R. Mann-Feder y M. Goyette (Eds.), Leaving care and the transition to adulthood: International contributions to theory, research, and practice (pp. 31-49). Oxford University Press. https://doi.org/10.1093/oso/9780190630485.003.0003

Hernández, R., Murillo, F. J. y Martínez-Garrido, C. (2014). Factores de ineficacia escolar. REICE. Revista Iberoamericana sobre Calidad, Eficacia y Cambio en Educación, 12(1), 103-118.

IGAXES. (2018). Memoría anual del año 2017. No publicado.

Jurado, P., Olmos, P. y Pérez, A. (2015). Los jóvenes en situación de vulnerabilidad y los programas formativos de transición al mundo del trabajo. Educar, 51(1), 211-224. https://doi.org/10.5565/rev/educar.648

Jussila, J. (2016). Elements of good institutional child welfare according to young people. En T. Suikkanen-Malin, M. Vestila y J. Jussila (Ed.), Foster care, childhood and E parenting in contemporary Europe (pp. 84-94). Publications of Kymenlaakso University of Applied Sciences.

Krichesky, G. J. y Murillo F. J. (2018). La colaboración docente como factor de aprendizaje y promotor de mejora. Un estudio de casos. Educación XX1, 21(1), 135-156. https://doi.org/10.5944/educxx 1.20181

Krinsky, M. (2010). A not so happy birthday: The foster youth transition from adolescence into adulthood. Family Court Review, 48, 250-254. https://doi.org/10.1111 
Martínez-Garrido, C. y Murillo, F. J. (2016). Investigación iberoamericana sobre enseñanza eficaz. Revista Mexicana de Investigación Educativa, 21(69), 47 1-499.

Melendro, M., De-Juanas, A. y Rodríguez, A. E. (2017). Déficits en la intervención socioeducativa con familias de adolescentes en riesgo de exclusión. Bordón, 69(1), 127-138. https://doi.org/10.13042/Bordon.2016.48596

Miles, S. y Singal, N. (2010). The education for all and inclusive education debate: Conflict, contradiction or opportunity? International Journal of Inclusive Education, 14(1), 1-15. https://doi.org/10.1080/13603110802265125

Montserrat, C., Casas, F., Malo, S. y Beltran, I. (2011). Los itinerarios educativos de los jóvenes extutelados. Ministerio de Sanidad y Política Social.

Murillo, F. J. (2005). La investigación sobre eficacia escolar. Octaedro.

Murillo, F. J. (2008). Enfoque, situación y desafíos de la investigación sobre eficacia escolar en América Latina y el Caribe. En R. Blanco (Dir.), Eficacia escolar y factores asociados en América Latina y el Caribe (pp. 17-48). UNESCO/LLECE

Murillo, F. J. y Krichesky, G. J. (2012). El proceso del cambio escolar. Una guía para impulsar y sostener la mejora de las escuelas. REICE. Revista Iberoamericana sobre Calidad, Eficacia y Cambio en Educación, 10(1), 26-43.

Murillo, F. J. y Martínez-Garrido, C. (2014). Las tareas para casa como recurso para una enseñanza de calidad. Revista de Psicología y Educación, 9(2), 31-44.

Murillo, F. J. y Román, M. (2011). ¿La escuela o la cuna? Evidencias sobre su aportación al rendimiento de los estudiantes de América Latina. Estudio multinivel sobre la estimación de los efectos escolares. Profesorado. Revista de Curriculum y Formación de Profesorado, 15(3), 27-53.

Okpych, N. J. y Courtney, M. E. (2014). Does education pay for youth formerly in foster care? Comparison of employment outcomes with a national sample. Children and Touth Services Review, 43(43), 18-28. https://doi.org/10.1016/j.childyouth.2014.04.013

Orellana-Fernández, R., Merellano-Navarro, E. y Almonacid-Fierro, A. (2018). Buen o buena docente de universidad: Perspectiva del personal directivo de carrera y de los mismos grupos docentes. Revista Electrónica Educare, 22(2), 1-27.

https://doi.org/10.15359/ree.22-2.6

Parrilla, A., Gallego, C. y Morriña, A. (2010). El complicado tránsito a la vida activa de jóvenes en riesgo de exclusión: Una perspectiva biográfica. Revista de Educación, 351, 211-233.

Protheroe, N. (2009). Good homework policy. Principal, 89, 42-45.

Save the Children. (2020). Covid-19: Cerrar la brecha. Impacto educativo y propuestas de equidad para la desescalada. Save de Children.

Sicilia, G. y Simancas, R. (2018). Equidad educativa en España: Comparación regional a partir de PISA 2015. Fundación Ramón Areces.

Simón, C., Barrios, A., Gutiérrez, H. y Muñoz, Y. (2020). Equidad, educación inclusiva y educación para la justicia social. ¿llevan todos los caminos a la misma meta? Revista Internacional de Educación para la Justicia Social, 8(2), 17-32. https://doi.org/10.15366/riejs2019.8.2.001

Stewart, C. J., Kum, H. C., Barth, R. P. y Duncan, D. F. (2014). Former foster youth: Employment outcomes up to age 30. Children and Touth Services Review, 36, 220-229. https://doi.org/10.1016/j.childyouth.2013.11.024 
Susinos, T. y Parrilla, A. (2013). Investigación inclusiva en tiempos difíciles. Certezas provisionales y debates pendientes. REICE. Revista Iberoamericana sobre Calidad, Eficacia y Cambio en Educación, 1 1(2), 87-98.

UNICEF. (2013). Inequality matters. Report on the world social situation. ONU.

Vinnerljung, B. y Hjern, A. (2011). Cognitive, educational and self-support outcomes of long-term foster care versus adoption. A Swedish national cohort study. Children and Touth Services Review, 33(10), 1902-1910. https://doi.org/10.1016/j.childyouth.2011.05.016

\section{Breve CV de los autores}

\section{Deibe Fernández-Simo}

Doctor en Ciencias de la Educación por la Universidad de Vigo. Actualmente es docente en el Departamento de Análisis e Intervención Psicosocioeducativa de la Universidad de Vigo. Su actividad investigadora se centra en adolescencia y juventud en dificultad social. En colaboración con los profesores Carrera Fernández y Cid Fernandez hemos abordado diversas temáticas relacionadas con la infancia y adolescencia en situación de riesgo social, siendo las publicaciones más recientes el artículo Déficits en la acción socioeducativa dirigida a adolescentes vulnerables latinoamericanos residentes en España (Revista Latinoamericana de Ciencias Sociales, Niñez y Juventud, 2020) y el libro sobre Adolescencias invisibles, (Andavira: Santiago de Compostela, 2020). ORCID ID: https://orcid.org/0000-0001-6202-4452. Email: jesfernandez@uvigo.es

\section{Xosé Manuel Cid Fernández}

Profesor titular de Teoría e Historia de la Educación en la Facultad de Ciencias de la Educación de la Universidade de Vigo. Su actividad investigadora se centra en Historia de la Educación en Galicia durante el siglo XX, así como en ámbitos de intervención de la Pedagogía Social, siendo miembro fundador de la Sociedad Iberoamericana de Pedagogía Social. En coautoría con la profesora carrera, ha participado de investigaciones y publicaciones sobre adolescencia, interculturalidad y pedagogía crítica y queer. En colaboración con el profesor Fernández Simo hemos abordado diversas temáticas relacionadas con la infancia y adolescencia en situación de riesgo social, siendo el más reciente el libro sobre Adolescencias invisibles, (Andavira: Santiago de Compostela, 2020). Es coordinador del Programa de Doctorado en Ciencias de la Educación y del Comportamiento de la Universidad de Vigo, desde su implantación en 2013, habiendo dirigido 15 tesis de temática histórica $\mathrm{y}$ pedagógico-social. ORDIC ID: https://orcid.org/0000-0002-7470-1737. Email: xcid@uvigo.es

\section{María Victoria Carrera-Fernández}

Docente en la Facultad de Ciencias de la Educación de la Universidade de Vigo. Su actividad investigadora se centra en la adolescencia, el bullying/ciberbullying desde una perspectiva socioecológica, la heteronormatividad y la pedagogía intercultural, crítica y queer. Entre sus últimas publicaciones destacan "Me and Us Versus the Others": Troubling the Bullying Phenomenon (Youth \& Society, 2019), Gender-bashing in Adolescents: Structural Relations with Heterosexual Matrix, Racism/Xenophobia and Attitudes toward Bullying (Journal of School Health, 2019), Patrolling the Boundaries of Gender: Beliefs, Attitudes and Behaviors Toward Trans and Gender Diverse People in 
Portuguese Adolescents (International Journal of Sexual Health, 2020) y Déficits en la acción socioeducativa dirigida a adolescentes vulnerables latinoamericanos residentes en España (Revista Latinoamericana de Ciencias Sociales, Niñez y Juventud, 2020). ORDIC ID: https://orcid.org/0000-0003-2 158-3084. Email: mavicarrera@uvigo.es 